\title{
Orbital Circularization in Binaries with A-Type Primary Stars
}

\author{
LYNN D. MATTHEWS \& ROBERT D. MATHIEU \\ Astronomy Dept., University of Wisconsin, Madison, WI 53706, USA
}

\section{INTRODUCTION}

It has long been recognized that tidal interaction between the components of close binaries will tend to circularize their orbits (e.g., Darwin 1879; Zahn 1977). In fact observations show an abundance of circular orbits in binaries with periods of days. However, the dissipation mechanism required for circularization, the timescales involved, and the dependence on period, mass, evolutionary state, and initial eccentricity of the system remain subjects of debate.

Circularization theories such as Zahn (1977) state that circularization in binaries where at least one component possesses a convective envelope is much more efficient than in binaries where both stars have radiative envelopes. Giuiricin et al. (1984; GMM) studied the period-eccentricity distribution of O-, B- and A-type binaries and argued that the observations were consistent with Zahn's theory for radiative envelopes. But recently, Tassoul (1988) has presented an alternative circularization theory with similarly efficient circularization in radiative- and convective-envelope binaries. In addition, period-eccentricity distributions for many samples of main-sequence convective-envelope binaries are now available. Here we reconsider the observed short-period eccentricity distribution of radiative-envelope binaries in light of these new observations and theory. Specifically, we investigate the period-eccentricity distribution of a sample of binaries with A-type primary stars.

\section{THE SAMPLE}

A set of 62 spectroscopic binaries with A-type primary components and orbital periods of less than $100^{d}$ has been selected from the Batten et al. (1989) catalogue of spectroscopic orbits. The sample has been restricted to those systems considered to have "good" or "excellent" determinations of the orbital elements. Several binaries with small $(e \leq 0.05)$ eccentricities were instead assigned $e=0$ based on the statistical analyses of Lucy \& Sweeney (1971). Eight binaries which were suspected to be either non-detached or to contain non-main-sequence components were omitted from the sample.

\section{THE PERIOD-ECCENTRICITY DISTRIBUTION}

The orbital period-eccentricity distribution for our sample of A-type binaries is shown in Figure 1. All binaries with orbital periods less than $P \approx 3^{d}$ have circular or nearly-circular orbits (i.e. $e<0.05$ ). Binaries with periods between $3-10^{d}$ are found with either circular or eccentric orbits, the maximum eccentricity increasing with period. Beyond $10^{d}$ all orbits are eccentric, although an 


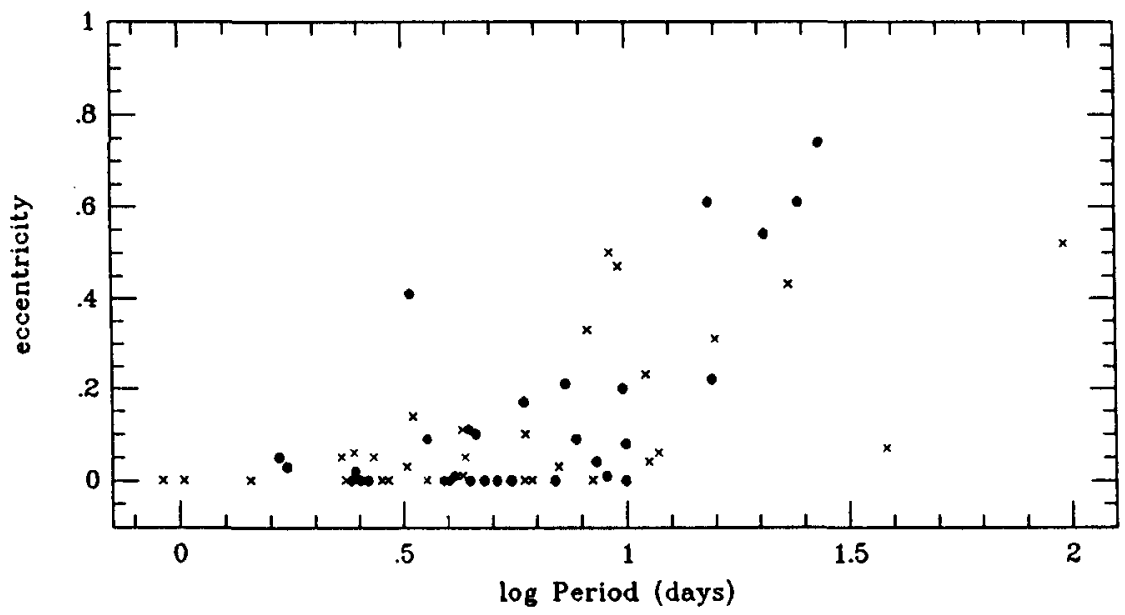

FIGURE 1. Period-eccentricity distribution for a sample of spectroscopic binaries with A-type primaries. Single-lined binaries are shown as crosses; double-lined binaries are shown as filled circles.

intriguing case exists at $\mathrm{P}=38.3^{d}$ with an eccentricity of only 0.07 . The longestperiod circular orbit is at $\mathrm{P}=9.9^{d}$. We note that there is a significant dearth of catalogued A-type spectroscopic binaries in the domain $\mathrm{P}=10-100^{d}$; hence there may be yet undiscovered systems with circular orbits at longer periods.

A circularization cutoff period $\left(\mathrm{P}_{\text {cut }}\right)$ in a homogeneous sample of binaries can be defined as the period of the longest-period circular orbit (Duquennoy et al. 1992). So defined, a cutoff period marks a lower limit on the longest period at which tidal circularization can be completed in the lifetime of a sample, and thus serves to indicate the efficacy of circularization in a given set of binaries. For the A-type binaries, such a cutoff is found at $P=9.9^{d}$ (Figure 1). Cutoff periods have previously been determined for several sets of convective-envelope binaries (see Table 1 in Mathieu et al. 1992). The cutoff period for our sample of A-type binaries is greater than the cutoff period found among convectiveenvelope binaries in the Hyades and Praesepe clusters (8.5 days; age $0.8 \mathrm{Gyr}$ ), comparable to that found for a sample of solar-mass field binaries (10.3 days), and less than that of the old open cluster M67 (12.4 days; age $4 \mathrm{Gyr}$ ).

\section{ANALYSIS}

The essential result of our study is that A-type binaries are found to have circular orbits at periods as long as 10 days, comparable to the longest-period circular orbits among older, solar-mass binaries. If convection in the stellar envelopes is critical to tidal circularization, and if circularization occurs primarily on the main sequence, then such long-period circular orbits in binaries having stars with radiative envelopes is unexpected.

In making comparisons, we should account for the larger masses and radii of the A-type stars relative to the solar-mass stars. In fact, if the nature of the stellar envelopes is not considered, approximate calculations (e.g., Mathieu \& Mazeh 1988, Eq. 7a) show that the differences in these parameters and in age 
can roughly account for the small differences in the cutoff period of the A-type binaries from those of the Hyades and M67.

The significance of the envelope for tidal circularization might also be explored by looking for variations in $\mathrm{P}_{\text {cut }}$ with secondary mass. Convective envelopes are found in main-sequence stars with $\mathcal{M} \leq 1.2 \mathcal{M}_{\odot}$; thus in our sample, double-lined systems have secondaries with radiative envelopes. Single-lined systems may have either convective- or radiative-envelope secondaries, the relative fraction depending on the secondary mass function. In our sample, both single-lined and double-lined systems are found with circular orbits at all periods less than $10^{d}$. Thus we find no evidence that the longest period circular orbits might be the result of more effective circularization by convectiveenvelope secondaries. Most significantly, the two longest period circular orbits are double-lined, each having two radiative-envelope stars (HD 82191, 9.0 ${ }^{d}$, Heard \& Hurkens 1973; HD 169691, 9.9 ${ }^{d}$, Popper 1969).

The evolutionary state of the binary components at the time that circularization occurs is a critical issue in this analysis due to the strong sensitivity of tidal circularization to stellar radius. Thus tidal circularization is more rapid in a binary having an evolved star compared with the main sequence rate. Since this increase in circularization rate has nothing to do with the radiative or convective nature of the stellar envelope per se, we would like to ensure that our sample does not include evolved stars. At present, detailed analyses of evolutionary state of fourteen of our double-lined binaries exist in the literature. Importantly, HD 39780 , with a near-circular orbit of $e=0.04$ at a period of $8.6^{d}$, is found by Andersen et al. (1987) to be near the zero-age main sequence $(t \approx 0.1$ Gyr). Thus the very low eccentricity of this system cannot be attributed to post-main-sequence evolution.

The youth of HD 39780 indicates that very small eccentricities can be present early in the lifetime of an A-type binary. GMM made the ad hoc suggestion that longer period circular orbits reflect a peak of $e \approx 0$ in the initial eccentricity distribution. The extent of tidal circularization during the premain-sequence phase has recently been recognized as an important issue. Zahn \& Bouchet (1989) argued that significant tidal circularization occurs during the pre-main-sequence phase in solar-mass binaries due to their large radii and deep convective zones, and also argued that the amount of circularization which takes place during the binary main-sequence lifetime is negligible. It is not clear yet whether observations support these conclusions (Mathieu et al. 1992). Nonetheless, similar arguments might be made for the more massive binaries considered here, recognizing though that their pre-main-sequence lifetimes are shorter than those of solar-mass stars and that their evolutionary histories differ (Palla \& Stahler 1990). Note that HD163708, the eccentric system at $\mathrm{P}=3.2828^{d}$ and $e=0.41$ (Andersen \& Gimémez 1985), and more generally the presence of eccentric orbits with periods below $P_{\text {cut }}$ remain to be explained in this hypothesis.

Under the hypothesis that main-sequence tidal circularization is the origin of all circular orbits among this sample of A-type binaries, our findings would suggest that the nature of the stellar envelope during this phase does not play a major role in setting the rate of tidal circularization. More specifically, the existence of A-type binaries with circular orbits at periods as long as $10^{d}$ is not 
consistent with the theoretical prediction of Zahn (1977) that circularization would not be effective in radiative-envelope binaries with periods longer than $\approx 2^{d}$. Furthermore, unlike the period-eccentricity distribution found by GMM for 0-, B- and A-type stars, there is no evidence in our more restricted sample for a marked increase in orbital eccentricities at $P \approx 2^{d}$. Indeed in our sample, with one exception, large eccentricities are first found at periods approaching the cutoff period. The source of this difference (for example sampling statistics in our smaller sample or the combination of a larger range of stellar masses in GMM) remains to be ascertained. Tassoul \& Tassoul (1992) have argued that effective main-sequence tidal circularization at long periods can be understood with the alternative circularization mechanism of Tassoul (1988).

If tidal circularization is effective in main-sequence binaries at periods as long as 10 days, the range of periods over which both circularized and eccentric orbits are found might be attributed in part to the range of ages in the sample. For example, Andersen \& Gimémez (1985) find HD 163708 to be young ( $t \approx 0.1-$ $0.2 \mathrm{Gyr}$ ), and hence propose that it may be only beginning to circularize its orbit. In addition, a range of initial eccentricities will produce a distribution of eccentric binaries near the cutoff period (Duquennoy et al. 1992). Finally, perturbations of a possible third body may account for small eccentricities in some of the systems regardless of their origin (Mazeh 1990; GMM).

Independent of whether the observed eccentricity distribution results from main-sequence or pre-main-sequence circularization, our comparisons reveal that both convective- and radiative-envelope binaries are able to achieve circularization at similar orbital periods before the end of their main-sequence lifetimes.

\section{CONCLUSION}

Binaries having A-type primaries with radiative envelopes have circular orbits with periods as long as $10^{d}$. This circularization cutoff period is comparable to cutoff periods found among older binaries with solar-mass, main-sequence primaries having convective envelopes. It is also significantly longer than predicted by traditional theories for main-sequence circularization of A-type binaries.

The longest period circular orbits cannot be attributed to post-mainsequence evolution. We suggest two possible explanations for the similarity of circularization cutoff periods in binaries with radiative- or convective-envelope primaries. First, tidal circularization occurs during the main-sequence phase at a rate which is not strongly dependent upon the nature of the stellar envelope. Second, tidal circularization is accomplished prior to the main sequence when the stars have larger radii and possibly convective envelopes. The two are not necessarily exclusive, as has been recognized in the study of solar-mass binaries (Duquennoy et al. 1992).

To summarize, prior to the end of their main-sequence lifetimes, binaries in which both components have radiative envelopes can achieve orbital circularization at periods as long as binaries in which one or both components are convective. We find no evidence that the nature of the main-sequence stellar envelope plays a critical role in the tidal circularization of main-sequence binaries. 


\section{REFERENCES}

Andersen, J., Clausen, J.V., \& Nordström, B. 1987, $A \& A, 175,60$

Andersen, J. \& Giménes, A. 1985, $A \& A, 130,102$

Batten, A.H., Fletcher, J.M., \& MacCarthy, D.G. 1989, Eighth Catalogue of the Orbital Elements of Spectroscopic Binaries (Victoria, Dominion Astrophys. Obs.), XVII

Darwin, G.H. 1879, Phil. Trans. Roy. Soc., 170, 1

Duquennoy, A., Mayor, M., \& Mermilliod, J.-C. 1992, in Binaries as Tracers of Stellar Formation, eds. A. Duquennoy \& M. Mayor, (Cambridge Press), in press

Giuricin, G., Mardirossian, F., \& Mezsetti, M. 1984, $A \& A$, 134, 365 (GMM)

Heard, J.F. \& Hurkens, R. 1973, J.R.A.S.C., 67, 306

Lucy, L.B. \& Sweeney, M.A. 1971, AJ, 76, 544

Mathieu, R.D., Duquennoy, A., Latham, D.W., Mayor, M., Maseh, T., \& Mermilliod, J.-C. 1992, in Binaries as Tracers of Stellar Formation, eds. A. Duquennoy \& M. Mayor, (Cambridge Press), in preas

Mathieu, R.D. \& Mareh, T. 1988, ApJ, 326, 256

Mazeh, T. 1990, $A J, 99,675$

Palla, F. \& Stahler, S.W. 1990, ApJ, 360, L47

Popper, D.M. 1969. PASP, 98, 1312

Tassoul, J.-L. 1988, $A p J, 324$, L71

Tassoul, J.-L. 1990, ApJ, 358, 196

Tassoul, J.-L. \& Tassoul, M. 1992, preprint

Zahn, J.-P. 1977, $A \& A, 57,383$

Zahn, J.-P. \& Bouchet, L. 1989, $A \& A, 223,112$ 